\title{
The Role of Microglia in Perioperative Neurocognitive Disorders
}

\author{
Wenguo Fan ${ }^{1,2 *}$, Lijia $\mathrm{Mai}^{2}$, Xiao Zhu ${ }^{3}$, Fang Huang ${ }^{2}$ and Hongwen $\mathrm{He}^{2 *}$ \\ ${ }^{1}$ Department of Anesthesiology, Guanghua School of Stomatology, Hospital of Stomatology, Sun Yat-sen University, \\ Guangzhou, China, ${ }^{2}$ Guangdong Provincial Key Laboratory of Stomatology, Guangzhou, China, ${ }^{3}$ The Marine Biomedical \\ Research Institute, Guangdong Medical University, Zhanjiang, China
}

\section{OPEN ACCESS}

Edited by:

Ukpong Bassey Eyo,

University of Virginia, United States

Reviewed by:

Adam Denes,

Institute of Experimental Medicine

(MTA), Hungary

Agnes Nadjar,

Université de Bordeaux, France

${ }^{*}$ Correspondence:

Wenguo Fan

fanweng@mail.sysu.edu.cn Hongwen $\mathrm{He}$

hehw@mail.sysu.edu.cn

Specialty section:

This article was submitted to

Non-Neuronal Cells,

a section of the journal

Frontiers in Cellular Neuroscience

Received: 22 May 2020

Accepted: 27 July 2020

Published: 18 August 2020

Citation:

Fan W, Mai L, Zhu X, Huang F and $\mathrm{He} \mathrm{H}$ (2020) The Role of Microglia in Perioperative

Neurocognitive Disorders.

Front. Cell. Neurosci. 14:261.

doi: 10.3389/fncel.2020.00261
Perioperative neurocognitive disorder (PND) is a common phenomenon associated with anesthesia and surgery and has been frequently described in the elderly and susceptible individuals. Microglia, which are the brain's major resident immune cells, play critical roles in maintaining neuronal homeostasis and synaptic plasticity. Accumulating evidence suggests microglial dysfunction occurring after anesthesia and surgery might perturb neuronal function and induce PND. This review aims to provide an overview of the involvement of microglia in PND to date. Possible cellular and molecular mechanisms regarding the connection between microglial activation and PND are discussed.

Keywords: perioperative neurocognitive disorder, microglia, neuroinflammation, anesthesia, surgery

\section{INTRODUCTION}

Microglia are a type of neuroglia occurring in the central nervous system (CNS) and can be defined as tissue-resident macrophages (Greter et al., 2015; Chowen and Garcia-Segura, 2020). They play important roles in the sustainment of normal physiological functions of CNS. A large amount of evidence indicates that microglia are involved in neuroinflammation as their activation and has been associated with many neurological disorders such as Alzheimer's disease (AD), neuropsychiatric disorders (Nayak et al., 2014).

Disordered neurocognitive function after surgery and anesthesia is a heterogeneous set of conditions, which includes any form of the acute event (postoperative delirium) and cognitive decline diagnosed up to 30 days after the procedure (delayed neurocognitive recovery) and up to 12 months (postoperative neurocognitive disorder, POCD; Evered et al., 2018; Safavynia and Goldstein, 2018). Previously, all forms of the impairment were called POCD, but more recently, perioperative neurocognitive disorders (PND) are recommended to be used as an overarching term for cognitive impairment identified in the perioperative period (Evered et al., 2018). PND is characterized as an acute or durable disturbance of cognitive functions including awareness, memory, attention, information processing, and cognitive flexibility (Hovens et al., 2012). The incidence of PND ranges from $8.9 \%$ to $46.1 \%$ depending on the study and type of surgery (Androsova et al., 2015). It occurs commonly in older patients (Monk et al., 2008; Evered et al., 2018). The pathogenesis of PND is multifaceted, which might be associated with anesthesia, tissue damage, neuroinflammation, surgical stress, psychological stress, and so on. In human studies, patients who develop PND showed cerebrospinal fluid (CSF) and plasma pro-inflammatory cytokines increases after anesthesia and surgery (Ji et al., 2013; Hirsch et al., 2016; Yuan et al., 2020). The more pronounced changes in CSF cytokines compared to plasma for several cytokines (MCP, MIP-1 $\alpha$, MIP-1 $\beta$ ) provide evidence for substantial inflammatory activity in the CNS (Hirsch et al., 2016). Since microglia are the macrophages of the CNS and play critical roles in neuroinflammatory disease (West et al., 2019), the significant alterations in some cytokines in CSF from patients indicate 
that microglia may be involved in PND in human (Helmy et al., 1999; Bromander et al., 2012; Hirsch et al., 2016; Yuan et al., 2020). Mounting evidence from animal studies suggests that microglia, like immune cells, are activated in the CNS and implicated in neuroinflammation and PND. This review aims to give an overview of the involvement of microglia in PND to date. Possible mechanisms regarding the connection between microglia and neuroinflammation in PND are discussed.

\section{MICROGLIA IN THE BRAIN}

The glial population in the CNS consists of microglia, oligodendrocytes, and astrocytes (Standring, 2016). The microglia account for between 5 and $12 \%$ of the total number of cells in the brain (Lawson et al., 1990). Adult microglia derive from primitive myeloid precursors that arise in the yolk sac early during embryonic development, after which they self-maintain locally and independently of blood-borne myeloid precursors (Greter et al., 2015). As their name suggests, microglia have a small cell body with highly branched processes in normal physiological conditions. As the resident macrophage cells, they act as immune sentinels in the CNS to sustain normal brain functions under healthy conditions. It is shown that microglia can be rapidly activated in a large number of pathological conditions such as inflammation. The activated microglia in the CNS are commonly referred to as M1-like or M2-like (Mosser and Edwards, 2008; Martinez and Gordon, 2014). The M1 microglia originally act to an insult and promote a proinflammatory response, while the M2 microglia are involved in tissue repair and remodeling and exert anti-inflammatory effect (Safavynia and Goldstein, 2018). However, the simple M1/M2 categories are challenged because there are many overlapping phenotypes with various functions and activation pathways in vivo studies in disease models (Colton et al., 2006; Martinez and Gordon, 2014; Heppner et al., 2015; Amici et al., 2017). Mounting evidence suggests that microglia not only are simply the brain's intrinsic immune cells but also are critical for neuronal circuit development, synaptic pruning, and brain homeostasis (Schafer et al., 2012; Zhan et al., 2014; Greter et al., 2015).

\section{MICROGLIAL ACTIVATION}

Microglia are the principal immune cells of the brain. As mentioned above, they react to modifications in the cellular environment through a graded response, in which any induced changes in morphology or gene/protein expression from the homeostatic state are termed activation or reactivity (LalancetteHébert et al., 2012; Greenhalgh et al., 2020). One of the characteristics of the activation is morphological changes and increased numbers of microglia (the latter is referred to as microgliosis). For example, surgery induces the microglial phenotype to a reactive hypertrophic cell body and shortened processes in the hippocampal region of aged animals (Terrando et al., 2016; Zhang et al., 2019). On the other hand, aged animals displayed cognitive impairment and microgliosis in the CA1 hippocampal region following surgery (Hovens et al., 2013;
Miller-Rhodes et al., 2019; Wang et al., 2019). Two possible mechanisms for microgliosis have been considered. First, it has been suggested that resident microglia proliferate (Inoue and Tsuda, 2018), but there is no study about microglia proliferation after anesthesia and surgery. Second, it has been proposed that bone-marrow-derived circulating monocytes may infiltrate into the hippocampus through the blood-brain barrier (BBB) and differentiate into microglia-like cells (Xu et al., 2014; Feng et al., 2017). Both microglia and astrocytes upregulate expression of chemokines such as monocyte chemoattractant protein 1 (MCP1/CCL2), which further facilitate monocyte recruitment into the hippocampus under inflammatory conditions (Xu et al., 2017). It remains to be elucidated whether such microgliosis following surgery relies on the local expansion of mature microglia or is achieved by infiltrating monocytes of blood. One study proposed that the infiltrating myeloid cells do not persist in the CNS after inflammation resolution and thus do not contribute to resident microglia (Ajami et al., 2011).

Due to the shared lineage of microglia and macrophages, many markers are common to both cell types. Mature microglia, similar to blood monocytes and other tissue-resident macrophages, express common markers such as the integrin CD11b, ionized calcium-binding adapter molecule 1 (Iba1), fractalkine receptor CX3CR1, Csf-1R and CD68 (Vizcaychipi et al., 2011; Hovens et al., 2013; Qiu et al., 2016; Feng et al., 2017). Thus, the microglia detected by immunostaining have not been thoroughly distinguished by their derivation. High throughput gene expression studies might identify the genes distinguishing microglia from other cell types in the CNS or in the periphery (Tay et al., 2017), which have identified surface markers and transcription factors specifically expressed by steady-state microglia but not by other macrophage populations or monocytes. These microglia-specific markers include $\mathrm{Fc}$ receptor-like S, purinergic receptor P2YG proteincoupled 12, sialic acid-binding immunoglobulin-type lectin $\mathrm{H}$, Tmem119 and Trem2 (Chiu et al., 2013; Butovsky et al., 2014; Bennett et al., 2016; Amici et al., 2017; Grassivaro et al., 2020). However, little is known about whether the microglia-specific surface markers and transcription factors alter their expression in neuroinflammation. Also, the molecular changes and functional difference between resident microglia and the monocyte-derived "microglia" remains ambiguous in PND.

Activated microglia are characterized by the changes in whole-genome expression and function in addition to morphological changes. Accumulating evidence indicates that anesthesia and surgery cause different degrees of microglial activation. The activation results in an inflammatory cascade promoting the synthesis and the secretion of inflammatory cytokines (IL-1 $\beta$, IL- 6 , and TNF- $\alpha$ ) and other inflammatory mediators (Buvanendran et al., 2006; Wang et al., 2019). Also, activated microglia recruit more blood monocytes (namely bone marrow-derived macrophages) into the CNS via reciprocal TNF- $\alpha$ expression (D'Mello et al., 2009). Neuroinflammation has become a key hallmark of neurological complications including PND (Spangenberg and Green, 2017; Subramaniyan and Terrando, 2019). The amplifying neuroinflammation and microglial activation could contribute to the development of 
PND (Hovens et al., 2013; Wang et al., 2015, 2016; Feng et al., 2017; Zhou X. et al., 2020). There is a limited amount of data about changes in microglia in the perioperative period in clinical studies. Non-invasive neuroimaging techniques may provide opportunities to assess the role of microglia directly (Tronel et al., 2017; Hierro-Bujalance et al., 2018). For example, microglial activation can be measured by positron emission tomography using uptake of $\left[{ }^{11} \mathrm{C}\right] \mathrm{PBR} 28$, which binds to the translocator protein, a protein upregulated in activated microglia and astrocytes (Datta et al., 2017; Forsberg et al., 2017; Albrecht et al., 2019; Werry et al., 2019). A recent clinical study demonstrated that patients showed a global downregulation of gray matter $\left[{ }^{11} \mathrm{C}\right]$ PBR28 binding in the early postoperative period, recovering or even increasing after 3 months. These processes may be related to post-surgical impairments of cognitive function (Forsberg et al., 2017). Depletion of microglia or interrupting microglial activation in hippocampus suppresses neuroinflammation and/or cognitive decline after surgery (Wan et al., 2014; Kawano et al., 2015; Li et al., 2016, 2018; Wang et al., 2016; Feng et al., 2017; Zhang et al., 2019; Wen et al., 2020; Zhou Y. et al., 2020), providing evidence that microglia may play critical roles in neuroinflammation and PND.

\section{HOW THE MICROGLIA ARE ACTIVATED}

\section{Systemic Inflammation Induced by Surgical Trauma}

Systemic inflammation can induce neuroinflammation and cognitive dysfunction in aged animals (Yamanaka et al., 2017; Huang et al., 2018). It is well-known that aseptic surgical trauma induces a systemic inflammatory response (Ni Choileain and Redmond, 2006). Damage-associated molecular patterns (DAMPs) are released by the damaged cells at the site of injury and promote and exacerbate the inflammatory response (Andersson and Tracey, 2011). Among the DAMPs, high mobility group box 1 (HMGB1) is the most studied as it has been described in preclinical models of cognitive impairment (Chavan et al., 2012; Li et al., 2013; Terrando et al., 2016). Increased levels of HMGB1 after surgery induce macrophage activation and the release of the pro-inflammatory cytokines (Terrando et al., 2016), which may induce an age-associated BBB dysfunction and increase its permeability (Yang et al., 2017). HMGB1 itself and these cytokines cross the $\mathrm{BBB}$ by diffusion or active transport causing macrophage migration into the hippocampus and microglia activation (Terrando et al., 2011, 2016). The activated microglia are the primary source of inflammatory cytokines that regulate microglia under feedback control (Hanisch, 2002). For example, following abdominal surgery under local anesthesia, the levels of TNF- $\alpha$, IL- 6 , and microglia activation are increased (Xu et al., 2014). Splenectomy performed under general anesthesia triggers a cognitive decline that may associate with proinflammatory cytokine-dependent activation of glial cells in the hippocampus (Wan et al., 2007). The peripherally produced cytokines can trigger neuroinflammation by activating microglia (Terrando et al., 2011; Hirsch et al., 2016), resulting in direct neurotoxicity and a cognitive decline following surgery.

\section{General Anesthetics}

The target organ of general anesthesia is the brain, but whether it is the main culprit causing cognitive decline by microglia remains controversial. The isoflurane or ketamine anesthesia causes morphological changes of microglia in rodents by using in vivo two-photon microscopy (Sun et al., 2019). It suggests that anesthetics may alter the function of microglia. Inhaled anesthetics have been demonstrated to cause neuroinflammation by activating microglia and may be involved in PND (Shen et al., 2013; Yan et al., 2016; QuirozPadilla et al., 2018; Wang et al., 2018). But a recent study showed that exposure to sevoflurane anesthesia for $8 \mathrm{~h}$ did not alter microglial activation in the adult monkey. The exposure had almost no effect on cognitive function (Walters et al., 2019). Etomidate, an intravenous anesthetic, induces PND attributed to hippocampal microglial activation during the early pathological stage (Li et al., 2020). But propofol, a widely used intravenous anesthetic, has no effects on neuroinflammation and cognition in the Alzheimer's transgenic model (Mardini et al., 2017). Another study showed that propofol-induced postoperative depressive-like behaviors, which is attributed to the inhibition of microglial activation (Song et al., 2019). in vitro studies show propofol has neuroprotective effects by attenuating inflammatory response in microglia (Gui et al., 2012; Peng et al., 2014). Also, propofol and other anesthetics have been demonstrated to possess neuroprotective effects (Matchett et al., 2009; Fan et al., 2015). These conflicting findings may be due to the anesthetic agent, concentration, duration of the exposures, methodological variation, and so on. Different anesthetics may modulate immune signaling pathways through different manners and show anti-inflammatory and proinflammatory effects in neuroinflammation. The effects of anesthetics on microglial activation in PND remain to be determined. The in vivo imaging in awake and anesthetized animals could help study microglia-neuron interactions (Liu Y. U. et al., 2019).

\section{Additional Mechanisms}

A recent study showed that peripheral surgery-induced CNS mast cell degranulation, which could trigger microglial activation and neuronal damage, contributing to PND (Zhang et al., 2016). Moreover, reactive oxygen species induced by nicotinamide adenine dinucleotide phosphate oxidase cause microglial activation that contributes to the neuroinflammation after the surgery (Qiu et al., 2016). Sirtuin-1 (SIRT1) is a member of the class III histone/protein deacetylase involved in the repression of inflammation (Kauppinen et al., 2013; Xie et al., 2013). SIRT1 activation inhibits nuclear factor kappa B signaling and enhances the resolution of inflammation (Kauppinen et al., 2013). Anesthesia and surgery inhibit hippocampal SIRT1 expression, resulting in microglial activation and an increase of proinflammatory cytokines in the hippocampus (Yan et al., 2019). Following tibial fracture surgery, the expression of CCL2 is upregulated in activated astrocytes. The astrocyte-derived CCL2 activates microglia participating in surgery-induced cognitive dysfunction and neuroinflammation (Xu et al., 2017). 


\section{ACTIVATED MICROGLIA ACT ON NEURONS}

Microglia mediate the inflammatory response in the hippocampus, resulting in the alteration of glutamatergic synaptic transmission and plasticity, which may underlie the behavioral comorbidities seen in patients (Riazi et al., 2015). The changes of inflammatory molecules in the brain following surgery and anesthesia may also directly bind receptors on neurons to alter neural actions and their normal adaptive roles. For example, TNF- $\alpha$ derived from glia mediates synaptic scaling via neuronal TNF receptors (Stellwagen and Malenka, 2006). A growing body of evidence indicates that in the aged brain, synaptic plasticity and memory show increased vulnerability to impairment by the IL-1 $\beta$ (Trompet et al., 2008; Patterson, 2015; Prieto et al., 2015). Other inflammatory mediators such as IL-6 and IL-9 are linked to physical and cognitive brain changes (McCarrey et al., 2014; Wharton et al., 2019). Electrophysiological recordings from CA1 hippocampal neurons revealed that PND mice exhibited impairment in AMPA receptor-mediated evoked excitatory postsynaptic currents (Wang et al., 2019). Astrocytes are the most abundant cell type in the CNS, which play a critical role in the formation and function of synapses. They also modulate neuronal excitability and plasticity (Greenhalgh et al., 2020). For example, hippocampal astrocyte dysfunction contributes to etomidate-induced long-lasting synaptic inhibition and cognitive dysfunction in older mice (Liu Y. et al., 2019). A subtype of reactive astrocytes, which are termed A1, is induced by activated microglia (Liddelow et al., 2017). A1 astrocytes contribute to the death of neurons and oligodendrocytes in neurodegenerative disorders (Liddelow et al., 2017). The A1-specific astrocyte activation is triggered by microglia during the initial pathological stage of PND and induces long-term synaptic inhibition and cognitive deficiencies (Li et al., 2020). Tau protein is primarily localized in CNS neurons and contributes to axonal integrity, whose tangles are strongly linked to neurodegeneration (Yang and Wang, 2018). The pathological mechanism of tau protein is associated with chronic neuroinflammatory processes, in which microglia play an important role (Vogels et al., 2019). The complement system is an important part of the

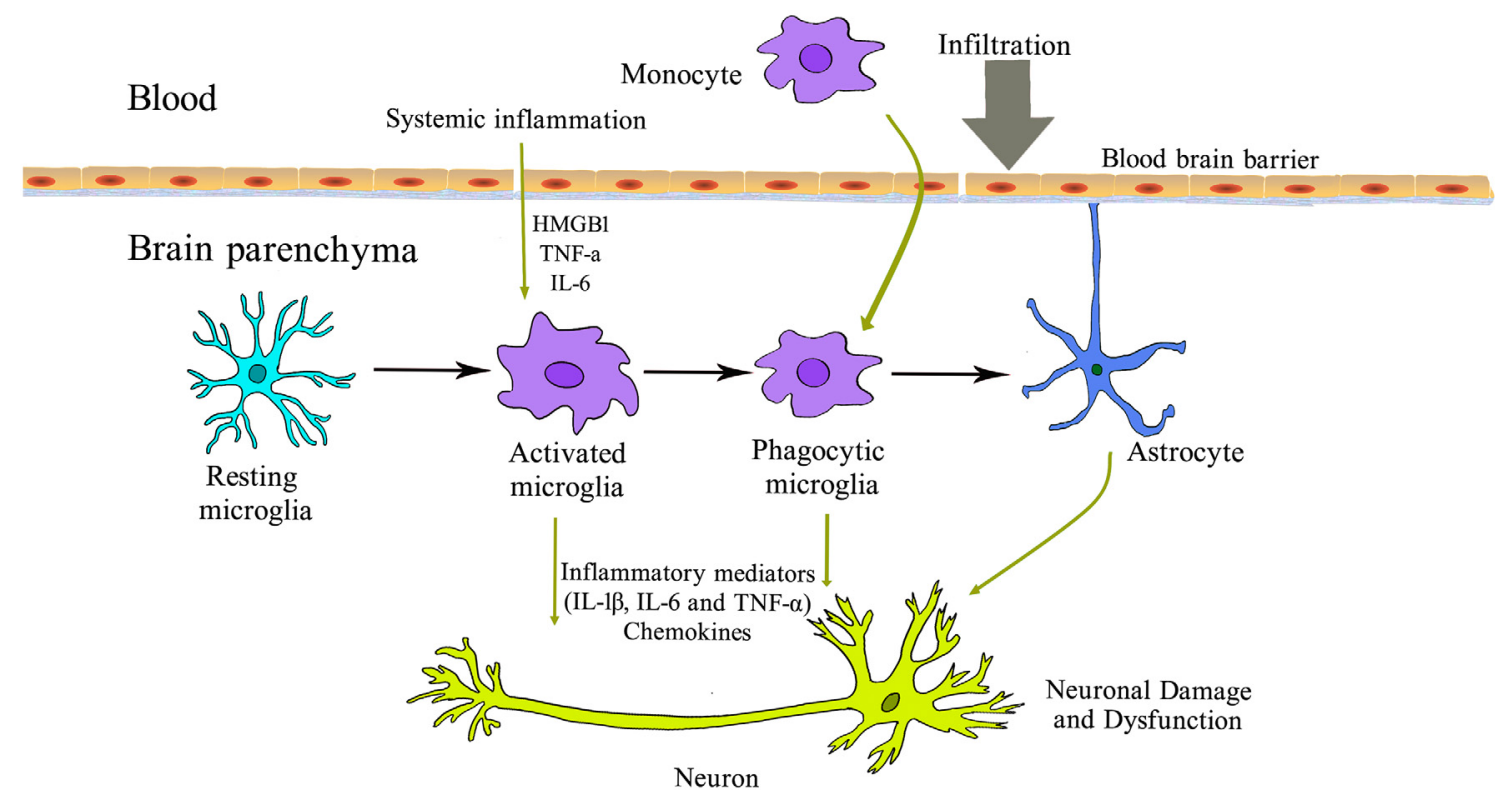

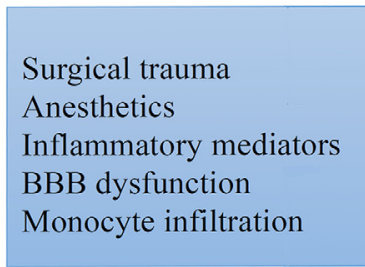

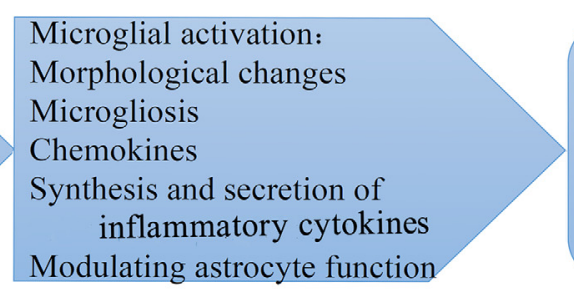

Neuron:

Synaptic inhibition

Changes of synaptic currents

Synaptic loss

Tau protein tangles

Death

FIGURE 1 | Schematic overview of microglial mechanisms involved in perioperative neurocognitive disorder (PND). Surgery and anesthesia induce a systemic inflammatory response. The increased pro-inflammatory mediators (cytokines, chemokines, alarmins, etc.) may compromise blood-brain barrier integrity, resulting in the infiltration of peripheral cells/factors into the brain parenchyma. Microglia are activated and initiate a cascade of inflammatory events that further activate other microglia and astrocyte. These processes contribute to neuronal damage and dysfunction and perioperative neurocognitive disorder. HMGB1, high-mobility group box 1 protein; IL, interleukin; TNF, tumor necrosis factor. 
innate immune system and involved in many neurological and neuropsychiatric diseases (Morgan, 2015). C3 levels and $\mathrm{C} 3 \mathrm{a}$ receptor expression are specifically increased in the hippocampus after surgery. The $\mathrm{C} 3 \mathrm{a}$ receptor activation contributes to neuroinflammation possibly through microglial activation, thereby resulting in the synaptic loss (Xiong et al., 2018). Taken together, these studies suggest that the neurons respond directly or indirectly to the inflammatory milieu induced by activated microglia and are influenced to affect cognitive changes. But the details of how activated microglia impair neurocognitive function after surgery warrants further research in the future.

\section{WHY ARE THE ELDERLY MORE VULNERABLE TO PND?}

As mentioned above, microglia play an important role in PND associated with neuroinflammation. PND is mainly seen in the elderly, and experimental studies also showed that it occurs frequently in old animals. Anesthesia and/or surgery did not cause a change in cognitive function in young adult mice (Zhao et al., 2016; Wang et al., 2018; Zhou X. et al., 2020). Hefendehl's study showed that compared to young mice, aged microglia mice showed different levels of morphological changes (Hefendehl et al., 2014). Moreover, in the normal physiological state, aged microglia have higher expression of pro-inflammatory genes and antigen-presenting markers, while anti-inflammatory cytokines and microglial activation inhibitory factors are down-regulated (Mosher and Wyss-Coray, 2014). The proinflammatory cytokines such as TNF- $\alpha$ and IL- $1 \beta$ released from hippocampal microglia isolated from aged rats following stimulation with lipopolysaccharide was significantly higher in comparison with young rats (Kawano et al., 2015). The shift of aged microglia tends to the proinflammatory' phenotypes (termed microglial priming) and may reflect an increase in inflammation associated with aging (Luo et al., 2010; Angelova and Brown, 2019). A recent study demonstrated that the hippocampal expression of SIRT1, which is associated with inflammation, decreased with age, resulting in microglial activation and increased proinflammatory cytokines in the hippocampus of aged rats. The trend of declining SIRT1 expression further deteriorated in aged rats after exposure to anesthesia and surgery (Yan et al., 2019). Moreover, the increased levels of NLRP3 expression in aged relative to young mice were observed in the hippocampus (Wang et al., 2018). The age-related morphological and functional changes in microglia may contribute to the susceptibility of the aging brain to dysfunction, often resulting in maladaptive responses, chronic inflammation,

\section{REFERENCES}

Ajami, B., Bennett, J. L., Krieger, C., McNagny, K. M., and Rossi, F. M. (2011). Infiltrating monocytes trigger EAE progression, but do not contribute to the resident microglia pool. Nat. Neurosci. 14, 1142-1149. doi: 10.1038/ nn. 2887

Albrecht, D. S., Forsberg, A., Sandstrom, A., Bergan, C., Kadetoff, D., Protsenko, E., et al. (2019). Brain glial activation in fibromyalgia - A multi-site and worsened outcomes after injury (Koellhoffer et al., 2017). Whether microglia are the primary players in PND is worthy to study further.

\section{CONCLUSIONS}

PND is a widespread phenomenon following the surgery and anesthesia and can have detrimental effects on an individual's quality of life and well-being. The pathogenesis of PND is not fully understood. Activation of microglia and neuro-glial interactions seem to be key mechanisms in PND (Figure 1). The precise mechanisms of microglia in PND so far have not been clear. Animal models have suggested that cognitive dysfunction is owing to neuroinflammation microglia involved, but clinical studies have not provided definite evidence on microglia involved in PND. Moreover, there are no clinical trials targeting microglia to lessen PND. Hopefully, there have been new tools to extensively characterize and interrogate complex microglia-neurons interactions in development and neurodegenerative disease, which include the generation of microglia in brain organoids (Ormel et al., 2018; Verheijen, 2019), massive single-cell sequencing datasets of microglia in health and disease (Mathys et al., 2017; Haage et al., 2019; Masuda et al., 2019; Van Hove et al., 2019), and a 3D triculture system (Park et al., 2018). Clinically, the innovation of PET and other neuroimaging techniques will improve our understanding of the microglial mechanism in PND. A better understanding of the role of microglia in PND could be helpful to treat patients more effectively in the perioperative period and find strategies target to microglia to prevent and/or treat PND in the elderly conditions.

\section{AUTHOR CONTRIBUTIONS}

WF designed and drafted the manuscript and figure. LM and $\mathrm{XZ}$ analyzed the data. $\mathrm{FH}$ and $\mathrm{HH}$ revised the manuscript. All authors contributed to the article and approved the submitted version.

\section{FUNDING}

This work was supported partly by the National Natural Science Foundation of China (Nos. 81771098 and 81541153).

\section{ACKNOWLEDGMENTS}

We are thankful to Miss Jin Shen for drawing the picture. doi: 10.1016/j.bbi.2018.09.018

Amici, S. A., Dong, J., and Guerau-de-Arellano, M. (2017). Molecular mechanisms modulating the phenotype of macrophages and microglia. Front. Immunol. 8:1520. doi: 10.3389/fimmu.2017.01520

Andersson, U., and Tracey, K. J. (2011). HMGB1 is a therapeutic target for sterile inflammation and infection. Annu. Rev. Immunol. 29, 139-162. doi: 10.1146/annurev-immunol-030409-101323 
Androsova, G., Krause, R., Winterer, G., and Schneider, R. (2015). Biomarkers of postoperative delirium and cognitive dysfunction. Front. Aging Neurosci. 7:112. doi: 10.3389/fnagi.2015.00112

Angelova, D. M., and Brown, D. R. (2019). Microglia and the aging brain: are senescent microglia the key to neurodegeneration? J. Neurochem. 151, 676-688. doi: $10.1111 /$ jnc. 14860

Bennett, M. L., Bennett, F. C., Liddelow, S. A., Ajami, B., Zamanian, J. L., Fernhoff, N. B., et al. (2016). New tools for studying microglia in the mouse and human CNS. Proc. Natl. Acad. Sci. U S A 113, E1738-E1746. doi: 10.1073/pnas. 1525528113

Bromander, S., Anckarsater, R., Kristiansson, M., Blennow, K., Zetterberg, H., Anckarsater, H., et al. (2012). Changes in serum and cerebrospinal fluid cytokines in response to non-neurological surgery: an observational study. J. Neuroinflammation 9:242. doi: 10.1186/1742-2094-9-242

Butovsky, O., Jedrychowski, M. P., Moore, C. S., Cialic, R., Lanser, A. J., Gabriely, G., et al. (2014). Identification of a unique TGF- $\beta$-dependent molecular and functional signature in microglia. Nat. Neurosci. 17, 131-143. doi: 10.1038/nn.3599

Buvanendran, A., Kroin, J. S., Berger, R. A., Hallab, N. J., Saha, C., Negrescu, C., et al. (2006). Upregulation of prostaglandin E2 and interleukins in the central nervous system and peripheral tissue during and after surgery in humans. Anesthesiology 104, 403-410. doi: 10.1097/00000542-200603000-00005

Chavan, S. S., Huerta, P. T., Robbiati, S., Valdes-Ferrer, S. I., Ochani, M., Dancho, M., et al. (2012). HMGB1 mediates cognitive impairment in sepsis survivors. Mol. Med. 18, 930-937. doi: 10.2119/molmed.2012.00195

Chiu, I. M., Morimoto, E. T., Goodarzi, H., Liao, J. T., O’Keeffe, S., Phatnani, H. P., et al. (2013). A neurodegeneration-specific gene-expression signature of acutely isolated microglia from an amyotrophic lateral sclerosis mouse model. Cell Rep. 4, 385-401. doi: 10.1016/j.celrep.2013.06.018

Chowen, J. A., and Garcia-Segura, L. M. (2020). Microglia, neurodegeneration and loss of neuroendocrine control. Prog. Neurobiol. 184:101720. doi: 10.1016/j. pneurobio.2019.101720

Colton, C. A., Mott, R. T., Sharpe, H., Xu, Q., Van Nostrand, W. E., and Vitek, M. P. (2006). Expression profiles for macrophage alternative activation genes in $\mathrm{AD}$ and in mouse models of AD. J. Neuroinflammation 3:27. doi: 10.1186/1742-2094-3-27

Datta, G., Violante, I. R., Scott, G., Zimmerman, K., Santos-Ribeiro, A., Rabiner, E. A., et al. (2017). Translocator positron-emission tomography and magnetic resonance spectroscopic imaging of brain glial cell activation in multiple sclerosis. Mult. Scler. 23, 1469-1478. doi: 10.1177/1352458516 681504

D’Mello, C., Le, T., and Swain, M. G. (2009). Cerebral microglia recruit monocytes into the brain in response to tumor necrosis factor $\alpha$ signaling during peripheral organ inflammation. J. Neurosci. 29, 2089-2102. doi: 10.1523/jneurosci.356708.2009

Evered, L., Silbert, B., Knopman, D. S., Scott, D. A., DeKosky, S. T., Rasmussen, L. S., et al. (2018). Recommendations for the nomenclature of cognitive change associated with anaesthesia and surgery-2018. Anesthesiology 129, 872-879. doi: 10.1097/ALN.0000000000002334

Fan, W., Zhu, X., Wu, L., Wu, Z., Li, D., Huang, F., et al. (2015). Propofol: an anesthetic possessing neuroprotective effects. Eur. Rev. Med. Pharmacol. Sci. 19, 1520-1529.

Feng, X., Valdearcos, M., Uchida, Y., Lutrin, D., Maze, M., and Koliwad, S. K. (2017). Microglia mediate postoperative hippocampal inflammation and cognitive decline in mice. JCI Insight 2:e91229. doi: 10.1172/jci.insight.91229

Forsberg, A., Cervenka, S., Jonsson Fagerlund, M., Rasmussen, L. S., Zetterberg, H., Erlandsson Harris, H., et al. (2017). The immune response of the human brain to abdominal surgery. Ann. Neurol. 81, 572-582. doi: 10.1002/ana.24909

Grassivaro, F., Menon, R., Acquaviva, M., Ottoboni, L., Ruffini, F., Bergamaschi, A., et al. (2020). Convergence between microglia and peripheral macrophages phenotype during development and neuroinflammation. J. Neurosci. 40, 784-795. doi: 10.1523/JNEUROSCI.1523-19.2019

Greenhalgh, A. D., David, S., and Bennett, F. C. (2020). Immune cell regulation of glia during CNS injury and disease. Nat. Rev. Neurosci. 21, 139-152. doi: 10.1038/s41583-020-0263-9

Greter, M., Lelios, I., and Croxford, A. L. (2015). Microglia versus myeloid cell nomenclature during brain inflammation. Front. Immunol. 6:249. doi: 10.3389/fimmu.2015.00249
Gui, B., Su, M., Chen, J., Jin, L., Wan, R., and Qian, Y. (2012). Neuroprotective effects of pretreatment with propofol in LPS-induced BV-2 microglia cells: role of TLR4 and GSK-3ß. Inflammation 35, 1632-1640. doi: 10.1007/s10753-0129478-x

Haage, V., Semtner, M., Vidal, R. O., Hernandez, D. P., Pong, W. W., Chen, Z., et al. (2019). Comprehensive gene expression meta-analysis identifies signature genes that distinguish microglia from peripheral monocytes/macrophages in health and glioma. Acta Neuropathol. Commun. 7:20. doi: 10.1186/s40478-0190665-y

Hanisch, U. K. (2002). Microglia as a source and target of cytokines. Glia 40, 140-155. doi: 10.1002/glia.10161

Hefendehl, J. K., Neher, J. J., Suhs, R. B., Kohsaka, S., Skodras, A., and Jucker, M. (2014). Homeostatic and injury-induced microglia behavior in the aging brain. Aging Cell 13, 60-69. doi: 10.1111/acel.12149

Helmy, S. A., Wahby, M. A., and El-Nawaway, M. (1999). The effect of anaesthesia and surgery on plasma cytokine production. Anaesthesia 54, 733-738. doi: 10.1046/j.1365-2044.1999.00947.x

Heppner, F. L., Ransohoff, R. M., and Becher, B. (2015). Immune attack: the role of inflammation in Alzheimer disease. Nat. Rev. Neurosci. 16, 358-372. doi: $10.1038 / \mathrm{nrn} 3880$

Hierro-Bujalance, C., Bacskai, B. J., and Garcia-Alloza, M. (2018). in vivo imaging of microglia with multiphoton microscopy. Front. Aging Neurosci. 10:218. doi: 10.3389/fnagi.2018.00218

Hirsch, J., Vacas, S., Terrando, N., Yuan, M., Sands, L. P., Kramer, J., et al. (2016). Perioperative cerebrospinal fluid and plasma inflammatory markers after orthopedic surgery. J. Neuroinflammation 13:211. doi: 10.1186/s12974016-0681-9

Hovens, I. B., Schoemaker, R. G., van der Zee, E. A., Heineman, E., Izaks, G. J., and van Leeuwen, B. L. (2012). Thinking through postoperative cognitive dysfunction: how to bridge the gap between clinical and pre-clinical perspectives. Brain Behav. Immun. 26, 1169-1179. doi: 10.1016/j.bbi.2012. 06.004

Hovens, I. B., Schoemaker, R. G., van der Zee, E. A., Heineman, E., Nyakas, C., and van Leeuwen, B. L. (2013). Surgery-induced behavioral changes in aged rats. Exp. Gerontol. 48, 1204-1211. doi: 10.1016/j.exger.2013.07.011

Huang, C., Irwin, M. G., Wong, G. T. C., and Chang, R. C. C. (2018). Evidence of the impact of systemic inflammation on neuroinflammation from a non-bacterial endotoxin animal model. J. Neuroinflammation 15:147. doi: 10.1186/s12974-018-1163-z

Inoue, K., and Tsuda, M. (2018). Microglia in neuropathic pain: cellular and molecular mechanisms and therapeutic potential. Nat. Rev. Neurosci. 19, 138-152. doi: 10.1038/nrn.2018.2

Ji, M. H., Yuan, H. M., Zhang, G. F., Li, X. M., Dong, L., Li, W. Y., et al. (2013). Changes in plasma and cerebrospinal fluid biomarkers in aged patients with early postoperative cognitive dysfunction following total hip-replacement surgery. J. Anesth. 27, 236-242. doi: 10.1007/s00540-012-1506-3

Kauppinen, A., Suuronen, T., Ojala, J., Kaarniranta, K., and Salminen, A. (2013). Antagonistic crosstalk between NF-кB and SIRT1 in the regulation of inflammation and metabolic disorders. Cell. Signal. 25, 1939-1948. doi: 10.1016/j.cellsig.2013.06.007

Kawano, T., Eguchi, S., Iwata, H., Tamura, T., Kumagai, N., and Yokoyama, M. (2015). Impact of preoperative environmental enrichment on prevention of development of cognitive impairment following abdominal surgery in a rat model. Anesthesiology 123, 160-170. doi: 10.1097/ALN.0000000000000697

Koellhoffer, E. C., McCullough, L. D., and Ritzel, R. M. (2017). Old maids: aging and its impact on microglia function. Int. J. Mol. Sci. 18:769. doi: 10.3390/ijms18040769

Lalancette-Hébert, M., Swarup, V., Beaulieu, J. M., Bohacek, I., Abdelhamid, E., Weng, Y. C., et al. (2012). Galectin-3 is required for resident microglia activation and proliferation in response to ischemic injury. J. Neurosci. 32, 10383-10395. doi: 10.1523/jneurosci.1498-12.2012

Lawson, L. J., Perry, V. H., Dri, P., and Gordon, S. (1990). Heterogeneity in the distribution and morphology of microglia in the normal adult mouse brain. Neuroscience 39, 151-170. doi: 10.1016/0306-4522(90)90229-w

Li, W., Chai, Q., Zhang, H., Ma, J., Xu, C., Dong, J., et al. (2018). High doses of minocycline may induce delayed activation of microglia in aged rats and thus cannot prevent postoperative cognitive dysfunction. J. Int. Med. Res. 46, 1404-1413. doi: 10.1177/0300060517754032 
Li, D., Chen, M., Meng, T., and Fei, J. (2020). Hippocampal microglial activation triggers a neurotoxic-specific astrocyte response and mediates etomidate-induced long-term synaptic inhibition. J. Neuroinflammation 17:109. doi: 10.1186/s12974-020-01799-0

Li, Y., Pan, K., Chen, L., Ning, J. L., Li, X., Yang, T., et al. (2016). Deferoxamine regulates neuroinflammation and iron homeostasis in a mouse model of postoperative cognitive dysfunction. J. Neuroinflammation 13:268. doi: 10.1186/s12974-016-0740-2

Li, R. L., Zhang, Z. Z., Peng, M., Wu, Y., Zhang, J. J., Wang, C. Y., et al. (2013). Postoperative impairment of cognitive function in old mice: a possible role for neuroinflammation mediated by HMGB1, S100B and RAGE. J. Surg. Res. 185, 815-824. doi: 10.1016/j.jss.2013.06.043

Liddelow, S. A., Guttenplan, K. A., Clarke, L. E., Bennett, F. C., Bohlen, C. J., Schirmer, L., et al. (2017). Neurotoxic reactive astrocytes are induced by activated microglia. Nature 541, 481-487. doi: 10.1038/nature21029

Liu, Y. U., Ying, Y., Li, Y., Eyo, U. B., Chen, T., Zheng, J., et al. (2019). Neuronal network activity controls microglial process surveillance in awake mice via norepinephrine signaling. Nat. Neurosci. 22, 1771-1781. doi: 10.1038/s41593019-0511-3

Liu, Y., Liu, W., Wang, X., Wan, Z., Gu, F., Ma, L., et al. (2019). Hippocampal astrocyte dysfunction contributes to etomidate-induced long-lasting synaptic inhibition. Biochem. Biophys. Res. Commun. 519, 803-811. doi: 10.1016/j.bbrc. 2019.09 .053

Luo, X. G., Ding, J. Q., and Chen, S. D. (2010). Microglia in the aging brain: relevance to neurodegeneration. Mol. Neurodegener. 5:12. doi: 10.1186/17501326-5-12

Mardini, F., Tang, J. X., Li, J. C., Arroliga, M. J., Eckenhoff, R. G., and Eckenhoff, M. F. (2017). Effects of propofol and surgery on neuropathology and cognition in the 3xTgAD Alzheimer transgenic mouse model. Br. J. Anaesth. 119, 472-480. doi: 10.1093/bja/aew397

Martinez, F. O., and Gordon, S. (2014). The M1 and M2 paradigm of macrophage activation: time for reassessment. F1000Prime Rep. 6:13. doi: 10.12703/p6-13

Masuda, T., Sankowski, R., Staszewski, O., Bottcher, C., Amann, L., and Sagar, et al. (2019). Spatial and temporal heterogeneity of mouse and human microglia at single-cell resolution. Nature 566, 388-392. doi: 10.1038/s41586-019-0924-x

Matchett, G. A., Allard, M. W., Martin, R. D., and Zhang, J. H. (2009). Neuroprotective effect of volatile anesthetic agents: molecular mechanisms. Neurol. Res. 31, 128-134. doi: 10.1179/174313209x393546

Mathys, H., Adaikkan, C., Gao, F., Young, J. Z., Manet, E., Hemberg, M., et al. (2017). Temporal tracking of microglia activation in neurodegeneration at single-cell resolution. Cell Rep. 21, 366-380. doi: 10.1016/j.celrep.2017. 09.039

McCarrey, A. C., Pacheco, J., Carlson, O. D., Egan, J. M., Thambisetty, M., An, Y., et al. (2014). Interleukin-6 is linked to longitudinal rates of cortical thinning in aging. Transl Neurosci. 5, 1-7. doi: 10.2478/s13380-014-0203-0

Miller-Rhodes, P., Kong, C., Baht, G. S., Saminathan, P., Rodriguiz, R. M., Wetsel, W. C., et al. (2019). The broad spectrum mixed-lineage kinase 3 inhibitor URMC-099 prevents acute microgliosis and cognitive decline in a mouse model of perioperative neurocognitive disorders. J. Neuroinflammation 16:193. doi: $10.1101 / 537472$

Monk, T. G., Weldon, B. C., Garvan, C. W., Dede, D. E., van der Aa, M. T., Heilman, K. M., et al. (2008). Predictors of cognitive dysfunction after major noncardiac surgery. Anesthesiology 108, 18-30. doi: 10.1097/01.anes. 0000296071.19434.1e

Morgan, B. P. (2015). The role of complement in neurological and neuropsychiatric diseases. Expert Rev. Clin. Immunol. 11, 1109-1119. doi: $10.1586 / 1744666 x .2015 .1074039$

Mosher, K. I., and Wyss-Coray, T. (2014). Microglial dysfunction in brain aging and Alzheimer's disease. Biochem. Pharmacol. 88, 594-604. doi: 10.1016/j.bcp. 2014.01.008

Mosser, D. M., and Edwards, J. P. (2008). Exploring the full spectrum of macrophage activation. Nat. Rev. Immunol. 8, 958-969. doi: 10.1038/nri2448

Nayak, D., Roth, T. L., and McGavern, D. B. (2014). Microglia development and function. Annu. Rev. Immunol. 32, 367-402. doi: 10.1146/annurev-immunol032713-120240

Ni Choileain, N., and Redmond, H. P. (2006). Cell response to surgery. Arch. Surg. 141, 1132-1140. doi: 10.1001/archsurg.141.11.1132
Ormel, P. R., Vieira de Sa, R., van Bodegraven, E. J., Karst, H., Harschnitz, O., Sneeboer, M. A. M., et al. (2018). Microglia innately develop within cerebral organoids. Nat. Commun. 9:4167. doi: 10.1038/s41467-018-06684-2

Park, J., Wetzel, I., Marriott, I., Dreau, D., D’Avanzo, C., Kim, D. Y., et al. (2018). A 3D human triculture system modeling neurodegeneration and neuroinflammation in Alzheimer's disease. Nat. Neurosci. 21, 941-951. doi: 10.1038/s41593-018-0175-4

Patterson, S. L. (2015). Immune dysregulation and cognitive vulnerability in the aging brain: Interactions of microglia, IL- $1 \beta, \mathrm{BDNF}$ and synaptic plasticity. Neuropharmacology 96, 11-18. doi: 10.1016/j.neuropharm.2014.12.020

Peng, M., Ye, J. S., Wang, Y. L., Chen, C., and Wang, C. Y. (2014). Posttreatment with propofol attenuates lipopolysaccharide-induced up-regulation of inflammatory molecules in primary microglia. Inflamm. Res. 63, 411-418. doi: 10.1007/s00011-014-0713-9

Prieto, G. A., Snigdha, S., Baglietto-Vargas, D., Smith, E. D., Berchtold, N. C., Tong, L., et al. (2015). Synapse-specific IL-1 receptor subunit reconfiguration augments vulnerability to IL-1 $\beta$ in the aged hippocampus. Proc. Natl. Acad. Sci. U S A 112, E5078-5087. doi: 10.1073/pnas.1514486112

Qiu, L. L., Ji, M. H., Zhang, H., Yang, J. J., Sun, X. R., Tang, H., et al. (2016). NADPH oxidase 2-derived reactive oxygen species in the hippocampus might contribute to microglial activation in postoperative cognitive dysfunction in aged mice. Brain Behav. Immun. 51, 109-118. doi: 10.1016/j.bbi.2015.08.002

Quiroz-Padilla, M. F., Guillazo-Blanch, G., Sanchez, M. Y., DominguezSanchez, M. A., and Gomez, R. M. (2018). Effects of excitotoxic lesion with inhaled anesthetics on nervous system cells of rodents. Curr. Pharm. Des. 24, 4-14. doi: 10.2174/1381612823666170817125015

Riazi, K., Galic, M. A., Kentner, A. C., Reid, A. Y., Sharkey, K. A., and Pittman, Q. J. (2015). Microglia-dependent alteration of glutamatergic synaptic transmission and plasticity in the hippocampus during peripheral inflammation. J. Neurosci. 35, 4942-4952. doi: 10.1523/jneurosci.4485-14.2015

Safavynia, S. A., and Goldstein, P. A. (2018). The role of neuroinflammation in postoperative cognitive dysfunction: moving from hypothesis to treatment. Front. Psychiatry 9:752. doi: 10.3389/fpsyt.2018.00752

Schafer, D. P., Lehrman, E. K., Kautzman, A. G., Koyama, R., Mardinly, A. R., Yamasaki, R., et al. (2012). Microglia sculpt postnatal neural circuits in an activity and complement-dependent manner. Neuron 74, 691-705. doi: 10.1016/j.neuron.2012.03.026

Shen, X., Dong, Y., Xu, Z., Wang, H., Miao, C., Soriano, S. G., et al. (2013). Selective anesthesia-induced neuroinflammation in developing mouse brain and cognitive impairment. Anesthesiology 118, 502-515. doi: 10.1097/aln. $0 \mathrm{~b} 013 \mathrm{e} 3182834 \mathrm{~d} 77$

Song, F., Lv, X., and Meng, J. (2019). Propofol induces postoperative depression and inhibits microglial function in mice. Mediators Inflamm. 2019:7651383. doi: 10.1155/2019/7651383

Spangenberg, E. E., and Green, K. N. (2017). Inflammation in Alzheimer's disease: Lessons learned from microglia-depletion models. Brain Behav. Immun. 61, 1-11. doi: 10.1016/j.bbi.2016.07.003

Standring, S. (2016). Gray's Anatomy. 41st Edn. London, UK: Elsevier Limited.

Stellwagen, D., and Malenka, R. C. (2006). Synaptic scaling mediated by glial TNFa. Nature 440, 1054-1059. doi: 10.1038/nature04671

Subramaniyan, S., and Terrando, N. (2019). Neuroinflammation and perioperative neurocognitive disorders. Anesth. Analg. 128, 781-788. doi: 10.1213/ANE. 0000000000004053

Sun, W., Suzuki, K., Toptunov, D., Stoyanov, S., Yuzaki, M., Khiroug, L., et al. (2019). in vivo two-photon imaging of anesthesia-specific alterations in microglial surveillance and photodamage-directed motility in mouse cortex. Front. Neurosci. 13:421. doi: 10.3389/fnins.2019.00421

Tay, T. L., Savage, J. C., Hui, C. W., Bisht, K., and Tremblay, M. E. (2017). Microglia across the lifespan: from origin to function in brain development, plasticity and cognition. J. Physiol. 595, 1929-1945. doi: 10.1113/jp272134

Terrando, N., Eriksson, L. I., Ryu, J. K., Yang, T., Monaco, C., Feldmann, M., et al. (2011). Resolving postoperative neuroinflammation and cognitive decline. Ann. Neurol. 70, 986-995. doi: 10.1002/ana.22664

Terrando, N., Yang, T., Wang, X., Fang, J., Cao, M., Andersson, U., et al. (2016). Systemic HMGB1 neutralization prevents postoperative neurocognitive dysfunction in aged rats. Front. Immunol. 7:441. doi: 10.3389/fimmu.2016. 00441 
Trompet, S., de Craen, A. J., Slagboom, P., Shepherd, J., Blauw, G. J., Murphy, M. B., et al. (2008). Genetic variation in the interleukin-1 $\beta$-converting enzyme associates with cognitive function. The PROSPER study. Brain 131, 1069-1077. doi: 10.1093/brain/awn023

Tronel, C., Largeau, B., Santiago Ribeiro, M. J., Guilloteau, D., Dupont, A. C., and Arlicot, N. (2017). Molecular targets for PET imaging of activated microglia: the current situation and future expectations. Int. J. Mol. Sci. 18:802. doi: $10.3390 /$ ijms 18040802

Van Hove, H., Martens, L., Scheyltjens, I., De Vlaminck, K., Pombo Antunes, A. R., De Prijck, S., et al. (2019). A single-cell atlas of mouse brain macrophages reveals unique transcriptional identities shaped by ontogeny and tissue environment. Nat. Neurosci. 22, 1021-1035. doi: 10.1038/s41593-019 $-0393-4$

Verheijen, B. M. (2019). Modeling brain somatic mosaicism with cerebral organoids, including a note on mutant microglia. Front. Mol. Neurosci. 12:277. doi: 10.3389/fnmol.2019.00277

Vizcaychipi, M. P., Xu, L., Barreto, G. E., Ma, D., Maze, M., and Giffard, R. G. (2011). Heat shock protein 72 overexpression prevents early postoperative memory decline after orthopedic surgery under general anesthesia in mice. Anesthesiology 114, 891-900. doi: 10.1097/aln.0b013e31820ad3ce

Vogels, T., Murgoci, A. N., and Hromadka, T. (2019). Intersection of pathological tau and microglia at the synapse. Acta Neuropathol. Commun. 7:109. doi: 10.1186/s40478-019-0754-y

Walters, J. L., Zhang, X., Talpos, J. C., Fogle, C. M., Li, M., Chelonis, J. J., et al. (2019). Sevoflurane exposure has minimal effect on cognitive function and does not alter microglial activation in adult monkeys. Neurotoxicology 71, 159-167. doi: 10.1016/j.neuro.2018.12.008

Wan, Q., Xu, L., and Bo, Y. (2014). Effects of Dexmedetomidine combined with Dezocine on cognition function and hippocampal microglia activation of rats. Int. J. Clin. Exp. Med. 7, 2787-2792.

Wan, Y., Xu, J., Ma, D., Zeng, Y., Cibelli, M., and Maze, M. (2007). Postoperative impairment of cognitive function in rats: a possible role for cytokinemediated inflammation in the hippocampus. Anesthesiology 106, 436-443. doi: 10.1097/00000542-200703000-00007

Wang, H. L., Liu, H., Xue, Z. G., Liao, Q. W., and Fang, H. (2016). Minocycline attenuates post-operative cognitive impairment in aged mice by inhibiting microglia activation. J. Cell Mol. Med. 20, 1632-1639. doi: 10.1111/jcmm. 12854

Wang, H. L., Ma, R. H., Fang, H., Xue, Z. G., and Liao, Q. W. (2015). Impaired spatial learning memory after isoflurane anesthesia or appendectomy in aged mice is associated with microglia activation. J. Cell Death 8, 9-19. doi: $10.4137 /$ jcd.s30596

Wang, Z., Meng, S., Cao, L., Chen, Y., Zuo, Z., and Peng, S. (2018). Critical role of NLRP3-caspase-1 pathway in age-dependent isoflurane-induced microglial inflammatory response and cognitive impairment. J. Neuroinflammation 15:109. doi: 10.1186/s12974-018-1137-1

Wang, T., Zhu, H., Hou, Y., Gu, W., Wu, H., Luan, Y., et al. (2019). Galantamine reversed early postoperative cognitive deficit via alleviating inflammation and enhancing synaptic transmission in mouse hippocampus. Eur. J. Pharmacol. 846, 63-72. doi: 10.1016/j.ejphar.2018.12.034

Wen, C., Xie, T., Pan, K., Deng, Y., Zhao, Z., Li, N., et al. (2020). Acetate attenuates perioperative neurocognitive disorders in aged mice. Aging 12, 3862-3879. doi: 10.18632/aging.102856

Werry, E. L., Bright, F. M., Piguet, O., Ittner, L. M., Halliday, G. M., Hodges, J. R., et al. (2019). Recent developments in TSPO PET imaging as a biomarker of neuroinflammation in neurodegenerative disorders. Int. J. Mol. Sci. 20:3161. doi: 10.3390/ijms20133161

West, P. K., Viengkhou, B., Campbell, I. L., and Hofer, M. J. (2019). Microglia responses to interleukin-6 and type I interferons in neuroinflammatory disease. Glia 67, 1821-1841. doi: 10.1002/glia.23634

Wharton, W., Kollhoff, A. L., Gangishetti, U., Verble, D. D., Upadhya, S., Zetterberg, H., et al. (2019). Interleukin 9 alterations linked to alzheimer disease in african americans. Ann. Neurol. 86, 407-418. doi: 10.1002/ana. 25543

Xie, J., Zhang, X., and Zhang, L. (2013). Negative regulation of inflammation by SIRT1. Pharmacol. Res. 67, 60-67. doi: 10.1016/j.phrs.2012.10.010
Xiong, C., Liu, J., Lin, D., Zhang, J., Terrando, N., and Wu, A. (2018). Complement activation contributes to perioperative neurocognitive disorders in mice. J. Neuroinflammation 15:254. doi: 10.1186/s12974-018-1292-4

Xu, J., Dong, H., Qian, Q., Zhang, X., Wang, Y., Jin, W., et al. (2017). Astrocytederived CCL2 participates in surgery-induced cognitive dysfunction and neuroinflammation via evoking microglia activation. Behav. Brain Res. 332, 145-153. doi: 10.1016/j.bbr.2017.05.066

Xu, Z., Dong, Y., Wang, H., Culley, D. J., Marcantonio, E. R., Crosby, G., et al. (2014). Peripheral surgical wounding and age-dependent neuroinflammation in mice. PLoS One 9:e96752. doi: 10.1371/journal.pone.0096752

Yamanaka, D., Kawano, T., Nishigaki, A., Aoyama, B., Tateiwa, H., ShigematsuLocatelli, M., et al. (2017). Preventive effects of dexmedetomidine on the development of cognitive dysfunction following systemic inflammation in aged rats. J. Anesth. 31, 25-35. doi: 10.1007/s00540-016-2264-4

Yan, N., Liu, Y., Liu, S., Cao, S., Wang, F., Wang, Z., et al. (2016). Fluoride-induced neuron apoptosis and expressions of inflammatory factors by activating microglia in rat brain. Mol. Neurobiol. 53, 4449-4460. doi: 10.1007/s12035-0159380-2

Yan, J., Luo, A., Gao, J., Tang, X., Zhao, Y., Zhou, B., et al. (2019). The role of SIRT1 in neuroinflammation and cognitive dysfunction in aged rats after anesthesia and surgery. Am. J. Transl. Res. 11, 1555-1568.

Yang, S., Gu, C., Mandeville, E. T., Dong, Y., Esposito, E., Zhang, Y., et al. (2017). Anesthesia and surgery impair blood-brain barrier and cognitive function in mice. Front. Immunol. 8:902. doi: 10.3389/fimmu.2017.00902

Yang, Y., and Wang, J. Z. (2018). Nature of tau-associated neurodegeneration and the molecular mechanisms. J. Alzheimers Dis. 62, 1305-1317. doi: 10.3233/jad170788

Yuan, Y., Li, Z., Yang, N., Han, Y., Ji, X., Han, D., et al. (2020). Exosome $\alpha-$ synuclein release in plasma may be associated with postoperative delirium in hip fracture patients. Front. Aging Neurosci. 12:67. doi: 10.3389/fnagi.2020. 00067

Zhan, Y., Paolicelli, R. C., Sforazzini, F., Weinhard, L., Bolasco, G., Pagani, F., et al. (2014). Deficient neuron-microglia signaling results in impaired functional brain connectivity and social behavior. Nat. Neurosci. 17, 400-406. doi: 10.1038/nn.3641

Zhang, X., Dong, H., Li, N., Zhang, S., Sun, J., Zhang, S., et al. (2016). Activated brain mast cells contribute to postoperative cognitive dysfunction by evoking microglia activation and neuronal apoptosis. J. Neuroinflammation 13:127. doi: 10.1186/s12974-016-0592-9

Zhang, D., Li, N., Wang, Y., Lu, W., Zhang, Y., Chen, Y., et al. (2019). Methane ameliorates post-operative cognitive dysfunction by inhibiting microglia NF$\kappa \mathrm{B} / \mathrm{MAPKs}$ pathway and promoting IL-10 expression in aged mice. Int. Immunopharmacol. 71, 52-60. doi: 10.1016/j.intimp.2019.03.003

Zhao, Y., Huang, L., Xu, H., Wu, G., Zhu, M., Tian, J., et al. (2016). Neuroinflammation induced by surgery does not impair the reference memory of young adult mice. Mediators Inflamm. 2016:3271579. doi: 10.1155/2016/3271579

Zhou, X., Lu, J., Wu, T., Jiang, X., Tian, W., Dai, W., et al. (2020). Multiple anesthesia/surgery cannot impair reference memory in adult mice. Mediators Inflamm. 2020:3736912. doi: 10.1155/2020/3736912

Zhou, Y., Wu, X., Ye, L., Bai, Y., Zhang, H., Xuan, Z., et al. (2020). Edaravone at high concentrations attenuates cognitive dysfunctions induced by abdominal surgery under general anesthesia in aged mice. Metab. Brain Dis. 35, 373-383. doi: 10.1007/s11011-019-00532-y

Conflict of Interest: The authors declare that the research was conducted in the absence of any commercial or financial relationships that could be construed as a potential conflict of interest.

Copyright (c) 2020 Fan, Mai, Zhu, Huang and He. This is an open-access article distributed under the terms of the Creative Commons Attribution License (CC BY). The use, distribution or reproduction in other forums is permitted, provided the original author(s) and the copyright owner(s) are credited and that the original publication in this journal is cited, in accordance with accepted academic practice. No use, distribution or reproduction is permitted which does not comply with these terms. 